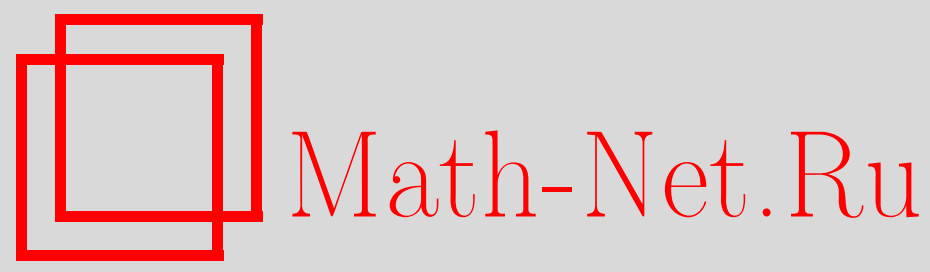

В. Б. Левенштам, Старшие приближения метода усреднения для квазилинейных параболических уравнений с быстро осциллирующей главной частью в случае задачи Коши, Матем. заметки, 1999, том 65, выпуск 4, 562-572

DOI: https://doi.org/10.4213/mzm1082

Использование Общероссийского математического портала Math-Net.Ru подразумевает, что вы прочитали и согласны с пользовательским соглашением http://www . mathnet.ru/rus/agreement

Параметры загрузки:

IP : 54.89 .56 .158

26 апреля 2023 г., 16:07:11

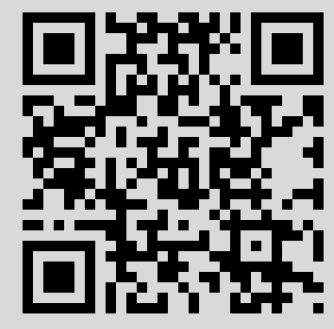




\section{СТАРШИЕ ПРИБЛИЖЕНИЯ МЕТОДА УСРЕДНЕНИЯ ДЛЯ КВАЗИЛИНЕЙНЫХ ПАРАБОЛИЧЕСКИХ УРАВНЕНИЙ С БЫСТРО ОСЦИЛЛИРУЮЩЕЙ ГЛАВНОЙ ЧАСТЬЮ В СЛУЧАЕ ЗАДАЧИ КОШИ}

\section{В.Б. Левенштам}

Предложен и обоснован алгоритм построения старших приближений метода усреднения для квазилинейных параболических уравнений с быстро осциллирующими коэффиициентами.

Библиография: 12 названий.

В работах [1], [2] обоснован метод усреднения для задачи об ограниченных на всей временной оси решениях квазилинейных параболических уравнений произвольного порядка $2 k$ с быстро осциллирующими коэффициентами при старших производных. Настоящая работа посвящена построению старших приближений метода усреднения для таких уравнений в случае задачи Коши. Предварительно сформулирована теорема 1 , в которой установлена однозначная разрешимость возмущенной задачи при больших значениях асимптотического параметра $\omega$ на том временном интервале $t \in[0, T]$, на котором разрешима усредненная задача, и доказана асимптотическая близость решений $u_{\omega}$ и $\stackrel{0}{v}$ этих задач. Далее возмущенная задача рассмотрена в предположении периодичности ее коэффициентов по “быстрому времени". Получена точная по параметру $\omega \gg 1$ оценка близости решений $u_{\omega}$ и $\stackrel{0}{v}$, а также при достаточной гладкости данных задачи построены старшие приближения $\stackrel{n}{u}_{\omega}, n=0,1, \ldots$, для которых при любых фиксированных неотрицательных числах $r$ и $s$ выполняются оценки

$$
\left\|u_{\omega}-\stackrel{n}{u}_{\omega}\right\|_{C^{s}\left([0, T], C^{r}\right)} \leqslant c_{n} \omega^{-(n+1)+s}
$$

где $\stackrel{0}{u_{\omega}}=\stackrel{0}{v}, c_{n}=c_{n}(r, s)=$ const. Через $C^{s}\left([0, T], C^{r}\right)$ обозначено пространство Гёльдера функций $u(x, t)$ гладкости $r$ по $x$ и гладкости $s$ по $t$ (определение см. ниже).

В работе существенно используются идеи И.Б. Симоненко [3], А.В. Васильевой, В.Ф. Бутузова [4], [5] и С.Д. Эйдельмана [6]. Для обыкновенных дифференциальных уравнений аналогичные схемы построения старших приближений изложены и обоснованы в работах В.В. Стрыгина [7] и Х. Т. Мовлянкулова, Л.В. Шаровой [8].

Работа выполнена при финансовой поддержке Российского фонда фундаментальных исследований, гранты № 96-01-01417, № 98-01-00136. 
По поводу асимптотического интегрирования различных уравнений математической физики с быстро осциллируюшими по пространственным переменным коэффициентами см. монографии [9]-[12] и имеющуюся там библиографию.

1. Пусть $k$ и $m$ - натуральные числа. Рассмотрим задачу Коши для квазилинейного параболического уравнения вида

$$
\begin{gathered}
\frac{\partial u}{\partial t}=\sum_{|\alpha|=2 k} a_{\alpha}\left(x, \delta^{2 k-1} u, t, \omega t\right) \mathscr{D}^{\alpha} u+f\left(x, \delta^{2 k-1} u, t, \omega t\right), \\
u(x, 0)=\varphi(x), \quad t \in[0, T], \quad T=\text { const }>0 .
\end{gathered}
$$

Здесь $x=\left(x_{1}, x_{2}, \ldots, x_{m}\right)$ - произвольная точка в арифметическом пространстве $\mathbb{R}^{m}$, $\omega$ - большой асимптотический параметр, $\alpha=\left(\alpha_{1}, \alpha_{2}, \ldots, \alpha_{m}\right)$ - мультииндекс,

$$
|\alpha|=\sum_{i=1}^{m} \alpha_{i}, \quad \mathscr{D}^{\alpha} u=\frac{\partial^{|\alpha|} u}{\partial x_{1}^{\alpha_{1}} \cdots \partial x_{m}^{\alpha_{m}}}
$$

$\delta^{2 k-1} u$ - вектор-функция, составленная из всевозможньх производных функции $u(x, t)$ по $x$ до порядка $2 k-1$ включительно. Обозначим количество таких производных чеpe3 $p$ :

$$
p=\sum_{i=0}^{2 k-1} \frac{(m+i-1) !}{i !(m-1) !}
$$

Пусть $M-$ произвольное ограниченное множество в пространстве $\mathbb{C}^{p} p$-мерных комплексных координатных векторов. (Под множеством $M$ можно подразумевать фиксированньй шар в $\mathbb{C}^{p}$, содержащий строго внутри себя все векторы $\left(\delta^{2 k-1} v\right)(x, t),(x, t) \in$ $\mathbb{R}^{m} \times[0, T]$, где $\stackrel{0}{v}-$ решение усредненной задачи, указанное ниже.)

Будем предполагать, что функции $a_{\alpha}(x, e, t, \tau), f(x, e, t, \tau)$ и их производные

$$
\frac{\partial a_{\alpha}}{\partial e_{i}}(x, e, t, \tau), \quad \frac{\partial f}{\partial e_{i}}(x, e, t, \tau)
$$

по компонентам вектора $e \in \mathbb{C}^{p}$ определены, непрерывны по совокупности переменных и равномерно ограничены при $x \in \mathbb{R}^{m}, e \in M, t \in[0, T], \tau \in[0, \infty)$. Кроме того, пусть для некоторого $\gamma_{0} \in(0,1)$ эти функции и их производные удовлетворяют по переменным $x, e, t, \tau$ условию Гёльдера с показателем $\gamma_{0}$ и константой $c(M)$, не зависящей от $(x, e, t, \tau) \in \mathbb{R}^{m} \times M \times[0, T] \times[0, \infty)$.

Предположим, что для уравнения (1) выполнено равномерное условие параболичности, т.е. для всех вещественных векторов $\sigma=\left(\sigma_{1}, \sigma_{2}, \ldots, \sigma_{m}\right)$ справедлива оценка

$$
(-1)^{k+1} \operatorname{Re} \sum_{|\alpha|=2 k} a_{\alpha}(x, e, t, \tau) \sigma^{\alpha} \geqslant \delta|\sigma|^{2 k},
$$

где постоянная $\delta=\delta(M)$ не зависит от $(x, e, t, \tau) \in \mathbb{R}^{m} \times M \times[0, T] \times[0, \infty)$. 
Пусть $a_{\alpha}$ и $f$ обладают средними $\stackrel{0}{a}{ }_{\alpha}$ и $\stackrel{0}{f}$ по последнему аргументу:

$$
\stackrel{0}{g}(x, e, t)=\lim _{N \rightarrow \infty} N^{-1} \int_{0}^{N} g(x, e, t, \tau) d \tau,
$$

где $g=a_{\alpha}$ или $f$, причем сходимость к пределу равномерна относительно $(x, e, t) \in \mathbb{R}^{m} \times$ $M \times[0, T]$.

Усредненная задача имеет вид

$$
\begin{gathered}
\frac{\partial v}{\partial t}=\sum_{|\alpha|=2 k} \stackrel{0}{a}_{\alpha}\left(x, \delta^{2 k-1} v, t\right) \mathscr{D}^{\alpha} v+\stackrel{0}{f}\left(x, \delta^{2 k-1} v, t\right), \\
v(x, 0)=\varphi(x), \quad t \in[0, T] .
\end{gathered}
$$

Все решения в этой работе понимаются в классическом смысле.

Введем некоторые обозначения. Пусть $r \geqslant 0,[r]$-целая часть $r$. Через $C^{r}:=C^{r}\left(\mathbb{R}^{m}\right)$ обозначим пространство непрерывно дифференцируемых до порядка $[r]$ включительно функций $u(x)$, определенных в $\mathbb{R}^{m}$ и удовлетворяющих при целых $r$ условию

$$
\|u\|_{C^{r}}=\sum_{|\alpha| \leqslant r} \sup _{x \in \mathbb{R}^{m}}\left|\mathscr{D}^{\alpha} u(r)\right|<\infty
$$

а при нецелых $r$ условию

$$
\|u\|_{C^{r}}=\|u\|_{C^{[r]}}+\sum_{|\alpha|=r} \sup _{x^{\prime \prime} \neq x^{\prime}}\left\{\left|\mathscr{D}^{\alpha} u\left(x^{\prime \prime}\right)-\mathscr{D}^{\alpha} u\left(x^{\prime}\right)\right| \cdot\left|x^{\prime \prime}-x^{\prime}\right|^{[r]-r}\right\}<\infty .
$$

Через $C^{\gamma}\left([0, T], C^{r}\right)$, где $r \geqslant 0, \gamma \in[0,1)$, обозначим банахово пространство непрерьвных в $\mathbb{R}^{m} \times[0, T]$ функций $u(x, t)$, имеющих непрерывные по совокупности переменных $(x, t)$ производные по $x$ до порядка $[r]$ включительно и удовлетворяющих при $\gamma=0$ условию

$$
\|u\|_{C^{0}\left([0, T], C^{r}\right)}=\sup _{t \in[0, T]}\|u(\cdot, t)\|_{C^{r}}<\infty
$$

а при $\gamma \in(0,1)$ условию

$$
\begin{aligned}
\|u\|_{C^{\gamma}\left([0, T], C^{r}\right)}= & \|u\|_{C^{0}\left([0, T], C^{r}\right)} \\
& +\sum_{|\alpha| \leqslant[r]} \sup _{\left(x, t^{\prime \prime}\right) \neq\left(x, t^{\prime}\right)}\left\{\left|\mathscr{D}^{\alpha} u\left(x, t^{\prime \prime}\right)-\mathscr{D}^{\alpha} u\left(x, t^{\prime}\right)\right| \cdot\left|t^{\prime \prime}-t^{\prime}\right|^{-\gamma}\right\}<\infty .
\end{aligned}
$$

Для функций $u(x, t, \tau)$, определенных и непрерьвных на множестве $\mathbb{R}^{m} \times[0, T] \times[0, \infty)$, аналогичньм образом введем норму $\|u\|_{C^{\gamma, \gamma_{1}}\left([0, T] \times[0, \infty), C^{r}\right)}$, где $r \geqslant 0, \gamma, \gamma_{1} \in[0,1)$. При $r \geqslant 0, s \geqslant 0$ определим норму

$$
\|u\|_{C^{s}\left([0, T], C^{r}\right)}=\sum_{i=0}^{[s]}\left\|\frac{\partial^{i}}{\partial t^{i}} u(x, t)\right\|_{C^{s-[s]}\left([0, T], C^{r}\right)}
$$


Аналогично, при $r \geqslant 0, s \geqslant 0, s_{1} \geqslant 0$ определим норму

$$
\|u\|_{C^{s, s_{1}}\left([0, T] \times[0, \infty), C^{r}\right)}=\sum_{i=0}^{[s]} \sum_{j=0}^{\left[s_{1}\right]}\left\|\frac{\partial^{i+j}}{\partial t^{i} \partial \tau^{j}} u(x, t, \tau)\right\|_{C^{s-[s], s_{1}-\left[s_{1}\right]}\left([0, T] \times[0, \infty), C^{r}\right)} .
$$

Предположим, что $\varphi \in C^{2 k+\gamma_{1}}, \gamma_{1} \in(0,1]$; задача $(3),(4)$ разрешима на отрезке времени $t \in[0, T]$ и решение $\stackrel{0}{v} \in C^{0}\left([0, T], C^{2 k-1+\gamma_{1}}\right)$.

Заметим, что из последнего предположения в силу известных оценок [6] следует принадлежность $\stackrel{0}{v}$ пространству $C^{\delta_{0} /(2 k)}\left([0, T), C^{2 k+\delta_{0}}\right)$, где $\delta_{0} \in\left(0, \min \left(\gamma_{0}, \gamma_{1}\right)\right)$.

Теорема 1. Существует число $\omega_{0}>0$ такое, что при $\omega>\omega_{0}$ задача (1), (2) однозначно разрешима на отрезке $t \in[0, T]$, причем для любого $\delta_{0} \in\left(0, \min \left(\gamma_{0}, \gamma_{1}\right)\right)$ выполнено соотношение

$$
\lim _{\omega \rightarrow \infty}\left\|u_{\omega}-\stackrel{0}{v}\right\|_{C^{\delta_{0} /(2 k)}\left([0, T], C^{\left.2 k+\delta_{0}\right)}\right.}=0
$$

где $u_{\omega} u \stackrel{0}{v}$-решения задач (1), (2) и (3), (4) соответственно.

Если функиии $a_{\alpha}(x, e, t, \tau)$ u $f(x, e, t, \tau)$ периодические по $\tau$ с одним и тем же периодом, то справедлива точная по параметру $\omega \gg 1$ оченка

$$
\left\|u_{\omega}-{ }^{0}\right\|_{C^{\delta_{0} /(2 k)}\left([0, T], C^{\left.2 k+\delta_{0}\right)}\right.} \leqslant c \omega^{\delta_{0} /(2 k)-1}, \quad c=\text { const } .
$$

Неулучшаемость указанной оценки подтверждается, например, задачей

$$
\frac{\partial u}{\partial t}=\Delta u+\sin \omega t, \quad u(x, 0)=0, \quad x \in \mathbb{R}^{m}
$$

Полное доказательство теоремы 1 мы здесь не приводим.

Для случая периодических по $\tau$ функций $a_{\alpha}$ и $f$ она фактически установлена в ходе доказательства теоремы 2 (см. ниже).

2. Пусть $l>0$. В дополнение к условиям п. 1 сделаем следующие предположения. Функции $a_{\alpha}(x, e, t, \tau),|\alpha|=2 k$, и $f(x, e, t, \tau)$ являются $l$-периодическими по $\tau$. Кроме того, существуют их непрерывные производные по всем аргументам любого порядка и при всех $(x, e, t, \tau) \in \mathbb{R}^{m} \times[0, T] \times[0, \infty)$ вьполняются оценки

$$
\left|\mathscr{D}_{x}^{\beta} \mathscr{D}_{e}^{\gamma} \frac{\partial^{i}}{\partial t^{i}} \frac{\partial^{i}}{\partial \tau^{i}} p(x, e, t, \tau)\right| \leqslant c_{\beta, \gamma, i}
$$

где $p=a_{\alpha}$ или $f, \beta=\left(\beta_{1}, \beta_{2}, \ldots, \beta_{m}\right), \gamma=\left(\gamma_{1}, \gamma_{2}, \ldots, \gamma_{p}\right)$ - произвольные мультииндексы, $i$ - произвольное целое неотрицательное число, $c_{\beta, \gamma, i}=$ const. Наконец, для любого $r \geqslant 0$ пусть $\varphi \in C^{r}$.

Введем следуюшие обозначения. Для любых $l$-периодических по $\tau$ функций $a(x, e, t, \tau)$ и $u(x, t, \tau)$ обозначим через $M_{\tau} a=\stackrel{0}{a}, M_{\tau} u=\stackrel{0}{u}$ их средние по $\tau$ (плавные 
составляющие), а через $R_{\tau} a=\stackrel{1}{a}, R_{\tau} u=\stackrel{1}{u}$ их быстро осциллирующие составляющие, так что $a=\stackrel{0}{a}+\stackrel{1}{a}, u=\stackrel{0}{u}+\stackrel{1}{u}$, где

$$
\stackrel{0}{a}(x, e, t)=l^{-1} \int_{0}^{l} a(x, e, t, \tau) d \tau, \quad \stackrel{0}{u}(x, t)=l^{-1} \int_{0}^{l} u(x, t, \tau) d \tau .
$$

Обозначая “быстрое время" $\omega t$ через $\tau$, перепишем задачу $(1),(2)$ в виде

$$
\begin{gathered}
\frac{\partial u}{\partial t}=\sum_{|\alpha|=2 k}\left(\stackrel{0}{a}_{\alpha}\left(x, \delta^{2 k-1} u, t\right)+\stackrel{1}{a}_{\alpha}\left(x, \delta^{2 k-1} u, t, \tau\right)\right) \mathscr{D}^{\alpha} u \\
+\stackrel{0}{f}\left(x, \delta^{2 k-1} u, t\right)+\stackrel{1}{f}\left(x, \delta^{2 k-1} u, t, \tau\right) \\
u(x, 0)=\varphi(x) .
\end{gathered}
$$

Решение задачи (6), (7) будем формально искать в виде ряда

$$
u(x, t, \tau)=\sum_{i=0}^{\infty} \omega^{-i} u_{i}(x, t, \tau)=\sum_{i=0}^{\infty} \omega^{-i}\left(\stackrel{0}{u}_{i}(x, t)+\stackrel{1}{u}_{i}(x, t, \tau)\right)
$$

Подставляя (8) в равенства (6), (7), предварительно заменив производную $\frac{\partial}{\partial t}$ в (6) на $\frac{\partial}{\partial t}+\omega \frac{\partial}{\partial \tau}$, раскладывая формально

$$
\begin{gathered}
\stackrel{0}{a}_{\alpha}\left(x, \delta^{2 k-1}\left(\sum_{i=0}^{\infty} \omega^{-i}\left(\stackrel{0}{u}_{i}(x, t)+\stackrel{1}{u}_{i}(x, t, \tau)\right)\right), t\right), \\
\stackrel{1}{a}_{\alpha}\left(x, \delta^{2 k-1}\left(\sum_{i=0}^{m} \omega^{-i}\left(\stackrel{0}{u}_{i}(x, t)+\stackrel{1}{u}_{i}(x, t, \tau)\right)\right), t, \tau\right), \\
\stackrel{0}{f(\cdots),}, \quad f(\cdots)
\end{gathered}
$$

в ряды Тейлора с центром разложения $\left(x, \delta^{2 k-1} \stackrel{0}{u}_{0}, t\right)$ и $\left(x, \delta^{2 k-1}{ }_{u_{0}}^{0}, t, \tau\right)$ соответственно, а затем группируя члены с одинаковыми степенями $\omega^{-1}$ отдельно для плавных и осциллирующих слагаемых, приходим к следующим задачам:

$$
\begin{aligned}
& \frac{\partial u_{0}^{1}}{\partial \tau}=0
\end{aligned}
$$

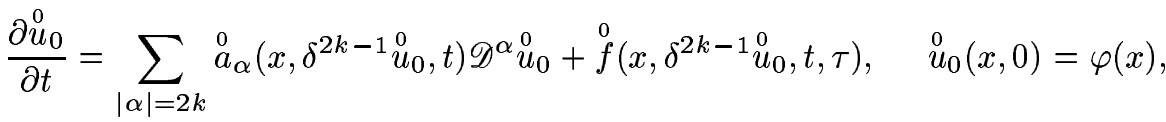

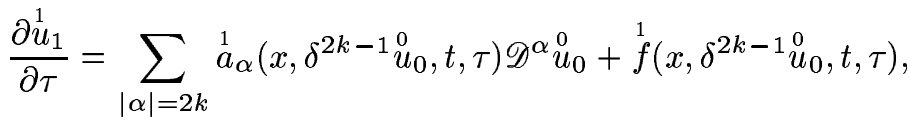




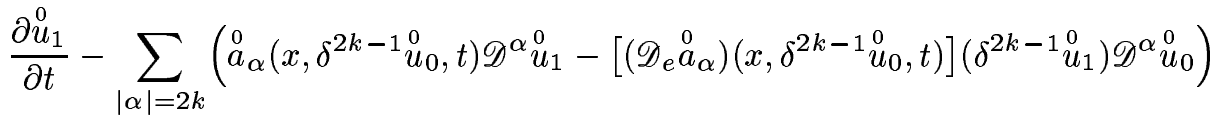

$$
\begin{aligned}
& -\left[\left(\mathscr{D}_{e}^{f} \stackrel{0}{f}\right)\left(x, \delta^{2 k-1}{ }^{0} u_{0}, t\right)\right]\left(\delta^{2 k-1}{ }_{u_{1}}^{0}\right)
\end{aligned}
$$

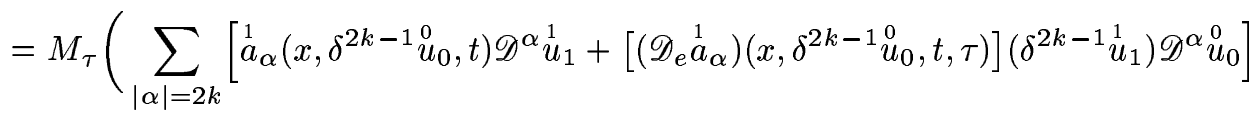

$$
\begin{aligned}
& \left.+\left[\left(\mathscr{D}_{e} f\right)\left(x, \delta^{2 k-1} \stackrel{0}{u_{0}}, t, \tau\right)\right]\left(\delta^{2 k-1} \stackrel{1}{u_{1}}\right)\right), \quad \stackrel{0}{u_{1}}(x, 0)=-\stackrel{1}{u}_{1}(x, 0,0),
\end{aligned}
$$

$$
\begin{aligned}
& \frac{\partial u_{n}^{1}}{\partial \tau}=\sum_{|\alpha|=2 k}\left(\stackrel{0}{a}_{\alpha}\left(x, \delta^{2 k-1} \stackrel{0}{u}_{0}, t\right) \mathscr{D}^{\alpha} \stackrel{1}{u}_{n-1}+\stackrel{1}{a}_{\alpha}\left(x, \delta^{2 k-1} \stackrel{0}{u}_{0}, t, \tau\right) \mathscr{D}^{\alpha} \stackrel{0}{u}_{n-1}\right.
\end{aligned}
$$

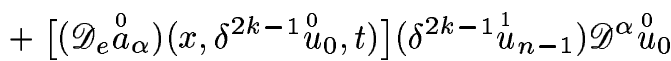

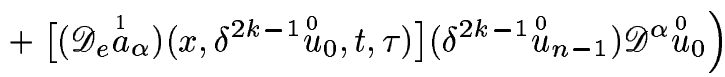

$$
\begin{aligned}
& +\left[\left(\mathscr{D}_{e} \stackrel{0}{f}\right)\left(x, \delta^{2 k-1} \stackrel{0}{u}_{0}, t\right)\right]\left(\delta^{2 k-1} \stackrel{1}{u}_{n-1}\right)+\left[\left(\mathscr{D}_{e} \stackrel{1}{f}\right)\left(x, \delta^{2 k-1} \stackrel{0}{u}_{0}, t, \tau\right)\right]\left(\delta^{2 k-1} \stackrel{0}{u}_{n-1}\right) \\
& +R_{\tau}\left(\sum _ { | \alpha | = 2 k } \left[\stackrel{1}{a}_{\alpha}\left(x, \delta^{2 k-1} \stackrel{0}{u}_{0}, t\right) \mathscr{D}^{\alpha} \stackrel{1}{u}_{n-1}\right.\right.
\end{aligned}
$$

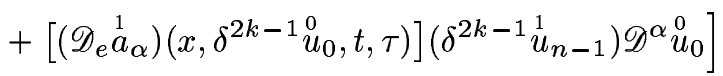

$$
\begin{aligned}
& \left.+\left[\left(\mathscr{D}_{e} f\right)\left(x, \delta^{2 k-1} \stackrel{0}{u}_{0}, t, \tau\right)\right]\left(\delta^{2 k-1} \stackrel{1}{u}_{n-1}\right)\right)-\frac{\partial \stackrel{1}{u}_{n-1}}{\partial t}+\stackrel{1}{S}_{n-2}(x, t, \tau), \\
& \frac{\partial \stackrel{0}{u}_{n}}{\partial t}-\sum_{|\alpha|=2 k}\left[\stackrel{0}{a}_{\alpha}\left(x, \delta^{2 k-1} \stackrel{0}{u}_{0}, t\right) \mathscr{D}^{\alpha} \stackrel{0}{u}_{n}-\left[\left(\mathscr{D}_{e} \stackrel{0}{a}_{\alpha}\right)\left(x, \delta^{2 k-1} \stackrel{0}{u_{0}}, t\right)\right]\left(\delta^{2 k-1} \stackrel{0}{u}_{n}\right) \mathscr{D}^{\alpha}{\stackrel{0}{u_{0}}}_{0}\right]
\end{aligned}
$$$$
-\left[\left(\mathscr{D}_{e} f\right)\left(x, \delta^{2 k-1} \stackrel{0}{u}_{0}, t\right)\right]\left(\delta^{2 k-1} \stackrel{0}{u}_{n}\right)
$$

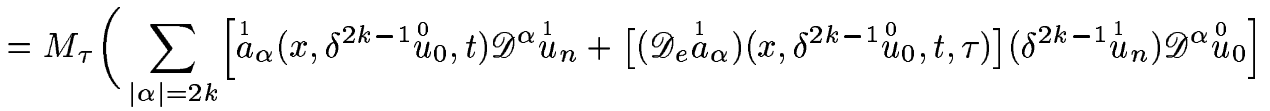$$
\left.+\left[\left(\mathscr{D}_{e} f\right)\left(x, \delta^{2 k-1} \stackrel{0}{u}_{0}, t, \tau\right)\right]\left(\delta^{2 k-1} \stackrel{1}{u}_{n}\right)\right)+\stackrel{0}{S}_{n-1}(x, t), \quad \stackrel{0}{u}_{n}(x, 0)=-\stackrel{1}{u}_{n}(x, 0,0) .
$$

Здесь через $\left[\left(\mathscr{D}_{e} a\right)\left(x, e_{0}, t, \tau\right)\right](e)$ обозначен частньй дифференциал функции $a$ по группе переменных $e$, т.е.

$$
\left[\left(\mathscr{D}_{e} a\right)\left(x, e_{0}, t, \tau\right)\right](e)=\sum_{i=0}^{p} \frac{\partial a}{\partial e_{i}}\left(x, e_{0}, t, \tau\right) e_{i}
$$

через $\stackrel{1}{S}_{n-2}(x, t, \tau)$ обозначена $l$-периодическая по $\tau$ функция, которая имеет по этому аргументу нулевое среднее и выражается через функции ${ }_{u}^{0}, u_{i}$ с номерами $i \leqslant n-2$; через $\stackrel{0}{S}_{n-1}(x, t)$ обозначена функция, которая выражается через $\stackrel{0}{u}_{i}, \stackrel{1}{u}_{i}$ с номерами $i \leqslant n-1$.

Отметим, что осциллируюшие составляющие $u_{k}$ определяются из обыкновенных дифференциальных уравнений $(9),(11),(13)$, в которых $t$ (как и $x$ ) интерпретируется 
в качестве параметра; следовательно, $\stackrel{1}{u}_{k}(x, t, \tau)$ должны удовлетворять таким уравнениям при всех значениях параметров $x \in \mathbb{R}^{m}, t \in[0, T]$.

Из уравнения (9) находим однозначно (функции $u_{k}$ имеют нулевое среднее по $\tau$ ) $\stackrel{1}{u_{0}}=0$. Задача Коши (10) совпадает с усредненной задачей $(3),(4)$ и по предположению имеет решение $\stackrel{0}{u}_{0}=\stackrel{0}{v}$. Многократно дифференцируя уравнение (10) по $x$ и $t$, применяя к производньм по $x$ решения известную априорную оценку для задачи Коши [6] и оценивая смешанные производные по $x, t$ в силу продифференцированных уравнений, получим для любых неотрищательных чисел $r$ и $s$ неравенства

$$
\|v\|_{C^{s}\left([0, T], C^{r}\right)} \leqslant \stackrel{0}{c}_{r, s}, \quad \stackrel{0}{c}_{r, s}=\text { const } .
$$

Обозначим правую часть равенства $(11)$ через $\varphi(x, t, \tau)$. Функция $\varphi$ имеет нулевое среднее по $\tau$, поэтому уравнение (11) однозначно разрешимо, при этом

$$
\stackrel{1}{u}_{1}(x, t, \tau)=\int_{0}^{\tau} \varphi(x, t, s) d s-l^{-1} \int_{0}^{l} d \tau \int_{0}^{\tau} \varphi(x, t, s) d s .
$$

Из линейной неоднородной задачи Коши (12) однозначно определяем функцию $\stackrel{0}{u}_{1}(x, t)$. Легко видеть, что для любых неотрищательных чисел $r, s, s_{1}$ справедливы оценки

$$
\left\|\stackrel{0}{u}_{1}\right\|_{C^{s}\left([0, T], C^{r}\right)} \leqslant \stackrel{1}{c}_{r, s}, \quad\left\|\stackrel{1}{u}_{1}\right\|_{C^{s, s_{1}}\left([0, T] \times[0, \infty), C^{r}\right)} \leqslant \stackrel{1}{d}_{r, s, s_{1}} .
$$

Предположим, что функции $\stackrel{0}{u}_{i}, \stackrel{1}{u}_{i}$ определены при всех $i \leqslant n-1$ и для них справедливы априорные оценки

$$
\left\|\stackrel{0}{u}_{i}\right\|_{C^{s}\left([0, T], C^{r}\right)} \leqslant \stackrel{i}{c}_{r, s}, \quad\left\|\stackrel{1}{u}_{i}\right\|_{C^{s, s_{1}}\left([0, T] \times[0, \infty), C^{r}\right)} \leqslant \stackrel{i}{d}_{r, s, s_{1}}
$$

где $\stackrel{i}{c}=$ const, $\stackrel{i}{d}=$ const. Тогда в силу тех же соображений, что использовались при рассмотрении задачи (11), из уравнения (13) однозначно найдем функцию $\stackrel{1}{u}_{n}(x, t, \tau)$. Тем самьм, задача Коши (14) единственным образом доставляет функцию $\stackrel{0}{u}_{n}(x, t)$. При этом справедливы оценки (15) для $i=n$.

Теперь обозначим $n$-ю частичную сумму ряда $(8)$ через $\stackrel{n}{u}(x, t, \tau)$ и положим $\stackrel{n}{u_{\omega}}(x, t)=$ $\stackrel{n}{u}(x, t, \omega t)$, т.е.

$$
\stackrel{n}{u}_{\omega}(x, t)=\sum_{i=0}^{n} \omega^{-i} u_{i}(x, t, \omega t)
$$

Справедлива

Теорема 2. Сущ,ествует $\omega_{0}>0$ mакое, что при $\omega>\omega_{0}$ для любых неотрииательных чисел $r$ и s при всех челых неотрицательных $n$ выполнены оченки

$$
\left\|u_{\omega}-\stackrel{n}{u}_{\omega}\right\|_{C^{s}\left([0, T], C^{r}\right)} \leqslant c_{n, r, s} \omega^{-(n+1)+s},
$$

где $u_{\omega}$ - указанное в теореме 1 решение задачи $(1),(2)$, a $c_{n, r, s}-$ не зависящая от $\omega$ постоянная.

Из приведенного ниже доказательства теоремы 2 вытекает следующее 
ЗАмЕЧАнИЕ. Если $r$ и $s$ - фиксированные неотрицательные числа, то для построения $n$-го приближения $\stackrel{n}{u_{\omega}}$ с оценкой $(16)$ достаточно выполнения следующих условий. $Ф$ ункция $\varphi \in C^{r_{1}}, r_{1}>r$. Функции $a_{\alpha}(x, e, t, \tau)$ и $f(x, e, t, \tau)$ являются $l$-периодическими по $\tau$ и удовлетворяют условиям п. 1. Кроме того, они имеют непрерьвные производные вида

$$
\mathscr{D}_{x}^{\beta} \mathscr{D}_{e}^{\lambda} \frac{\partial^{i}}{\partial t^{i}} \frac{\partial^{i_{1}}}{\partial \tau^{i_{1}}}
$$

где $|\beta| \leqslant k_{0}=\max (0,[r]-2 k), i+i_{1} \leqslant k_{1}=\max (0,[s]-1),|\lambda| \leqslant n+1+k_{0}+k_{1}$, причем эти производные удовлетворяют условию Гёльдера по совокупности переменных $x, e, t, \tau$ с показателем $\alpha_{1}>\{r\}, \alpha_{2}=\max \left(\alpha_{1},\{s\}\right), \alpha_{3}=\alpha_{4}=\{s\}$ соответственно и константами, не зависящими от $(x, e, t, \tau) \in \mathbb{R}^{m} \times M \times[0, T] \times[0, \infty)$. Здесь $M$ - то же множество, что и выше, а $\{b\}$ - дробная часть числа $b$.

ДОКАЗАТЕЛЬСТВО ТЕОРЕМЫ 2. Пусть

$$
\stackrel{n}{u}_{\omega}^{\prime}(x, t)=\stackrel{n}{u}_{\omega}(x, t)+\omega^{-(n+1)} \stackrel{1}{u}_{n+1}(x, t, \omega t)-\omega^{-(n+1)} \stackrel{1}{u}_{n+1}(x, 0,0)
$$

Очевидно, оценка (16) эквивалентна такой же оценке для разности $u_{\omega}-\stackrel{n}{u_{\omega}^{\prime}}:=w_{\omega}$ (далее индекс $\omega$ будем, как правило, опускать). Подставляя $u=\stackrel{n}{u}^{\prime}+w$ в равенства $(1),(2)$ и учитьвая соотношения (9)-(14), получим следующую задачу Коши для $w$ :

$$
\begin{aligned}
& \frac{\partial w}{\partial t}-\sum_{|\alpha|=2 k}\left(a_{\alpha}\left(x, \delta^{2 k-1}\left(\stackrel{n}{u}^{\prime}+w\right), t, \omega t\right) \mathscr{D}^{\alpha} w-\left[\left(\mathscr{D}_{e} \stackrel{0}{a}_{\alpha}\right)\left(x, \delta^{2 k-1}{ }_{v}^{0}, t\right)\right]\left(\delta^{2 k-1} w\right) \mathscr{D}^{\alpha}{ }^{0}\right) \\
& -\left[\left(\mathscr{D}_{e} f\right)\left(x, \delta^{2 k-1}{ }_{v}^{0}, t\right)\right]\left(\delta^{2 k-1} w\right) \\
& =\sum_{|\alpha|=2 k}\left(\left[a_{\alpha}\left(x, \delta^{2 k-1}\left(\stackrel{n}{\prime}^{\prime}+w\right), t, \omega t\right)-a_{\alpha}\left(x, \delta^{2 k-1} \stackrel{n}{u}^{\prime}, t, \omega t\right)\right] \mathscr{D}^{\alpha} \stackrel{n}{u}^{\prime}\right. \\
& \left.-\left[\left(\mathscr{D}_{e} \stackrel{0}{a}_{\alpha}\right)\left(x, \delta^{2 k-1}{ }^{0}, t\right)\right]\left(\delta^{2 k-1} w\right) \mathscr{D}^{\alpha}{ }^{0}\right) \\
& +f\left(x, \delta^{2 k-1}\left(\stackrel{n}{u^{\prime}}+w\right), t, \omega t\right)-f\left(x, \delta^{2 k-1} \stackrel{n}{u}^{\prime}, t, \omega t\right) \\
& -\left[\left(\mathscr{D}_{e} f\right)\left(x, \delta^{2 k-1} v^{0}, t\right)\right]\left(\delta^{2 k-1} w\right)+\omega^{-(n+1)} a(x, t, \omega t, \omega):=\Phi(x, w, \omega t, \omega), \\
& w(x, 0)=0 .
\end{aligned}
$$

Здесь $a(x, t, \tau, \omega)$ - бесконечно дифференцируемая по $(x, t, \tau) \in \mathbb{R}^{m} \times[0, T] \times[0, \infty)$ при $\omega>0$ функция, причем для любых неотрицательных чисел $r, s, s_{1}$ справедлива оценка

$$
\|a(x, t, \tau, \omega)\|_{C^{s, s_{1}}\left([0, T] \times[0, \infty), C^{r}\right)} \leqslant c_{r, s, s_{1}},
$$

где постоянная $c_{r, s, s_{1}}$ не зависит от $\omega>1$. Это легко устанавливается с помощью известной оценки остаточного члена ряда Тейлора и неравенства (5). 
Обозначим через $G_{w}^{\omega}(t, \tau, x, \xi)$ фундаментальное решение уравнения

$$
\begin{aligned}
\frac{\partial u}{\partial t} & -\sum_{|\alpha|=2 k}\left(a_{\alpha}\left(x, \delta^{2 k-1}\left(u^{\prime}+w\right), t, \omega t\right) \mathscr{D}^{\alpha} u-\left[\left(\mathscr{D}_{e}{ }^{\stackrel{0}{\alpha}}\right)\left(x, \delta^{2 k-1}{ }_{v}^{0}, t\right)\right]\left(\delta^{2 k-1} w\right) \mathscr{D}^{\alpha} v^{0}\right) \\
& -\left[\left(\mathscr{D}_{e} f\right)\left(x, \delta^{2 k-1}{ }^{0}, t\right)\right]\left(\delta^{2 k-1} u\right)=0 .
\end{aligned}
$$

Тогда решение $w$ задачи $(1),(2)$ является неподвижной точкой действующего в пространстве $C^{\gamma}\left([0, T], C^{2 k+\gamma}\right), \gamma \in(0,1)$, оператора $\mathscr{P}_{\omega}$ :

$$
\mathscr{P}_{\omega}(w)=\int_{0}^{t} d \tau \int_{\mathbb{R}^{m}} G_{w}^{\omega}(t, \tau, x, \xi) \Phi(\xi, w(\xi, \tau), \omega \tau, \omega) d \xi
$$

Докажем, что при $\omega \gg 1$ оператор $\mathscr{P}_{\omega}$ в некотором шаре пространства $C^{\gamma}([0, T]$, $\left.C^{2 k+\gamma}\right)$ с центром в нуле радиуса $r_{\omega}=O\left(\omega^{-(n+1)}\right)$ является сжатием. Для этого нам потребуются некоторые (приведенные в [2]) оценки функции $G_{w}^{\omega}(t, \tau, x, \xi)[6]$.

Введем некоторые обозначения. Пусть $h \in \mathbb{R}^{m}, h^{1} \geqslant 0$. Тогда

$$
\begin{gathered}
\Delta \chi(x, t):=\chi\left(x+h, t+h^{1}\right)-\chi(x, t), \\
v(x, t, \psi)=\int_{t_{0}}^{t} d \tau \int_{\mathbb{R}^{m}} G_{w}^{\omega}(t, \tau, x, \xi) \psi(\xi, \tau) d \xi, \quad 0 \leqslant t_{0} \leqslant t \leqslant t+h^{1} \leqslant T .
\end{gathered}
$$

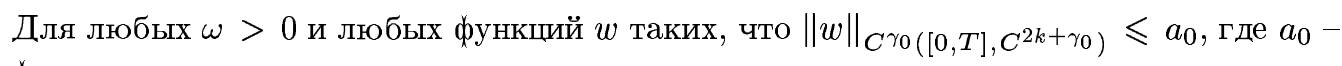
фиксированная постоянная, справедливы следующие оценки:

$$
\begin{aligned}
\left|\mathscr{D}_{x}^{\alpha} G_{w}^{\omega}(t, \tau, x, \xi)\right| \leqslant & c_{0}(t-\tau)^{-(m+|\alpha|) /(2 k)} e^{-c_{1} \rho(t, \tau, x, \xi)}, \\
\left|\Delta \mathscr{D}_{x}^{\alpha} G_{w}(t, \tau, x, \xi)\right| \leqslant & c_{0}(t-\tau)^{-\left(m+|\alpha|+\gamma^{\prime}\right) /(2 k)} \\
& \times \max \left(e^{-c_{1} \rho(t, \tau, x, \xi)}, e^{-c_{1} \rho(t, \tau, x+h, \xi)}\right),
\end{aligned}
$$

если $0 \leqslant h^{1} \leqslant b(t-\tau)$ при некоторой постоянной $b>0$,

$$
\left|\Delta \mathscr{D}_{x}^{\alpha} v_{\omega}\right| \leqslant c_{0}\left(|h|^{\gamma^{\prime}}+\left|h^{1}\right|^{\gamma^{\prime} /(2 k)}\right)\left(t-t_{0}\right)^{\left(2 k-|\alpha|+\gamma_{0}-\gamma^{\prime}\right) /(2 k)}\|\psi\|_{C^{0}\left([0, T], C^{\gamma_{0}}\right)}
$$

где

$$
\rho(t, \tau, x, \xi)=\sum_{i=1}^{m}\left(\left|x_{i}-\xi_{i}\right|(t-\tau)^{-1 /(2 k)}\right)^{2 k /(2 k-1)}
$$

$c_{0}, c_{1}$ - положительные постоянные, которые не зависят от $\omega, w, t_{0}, t, x, \xi, h, h^{1}$ и $\psi$, $0<\gamma^{\prime} \leqslant \gamma_{0}$, причем $\gamma^{\prime}<\gamma_{0}$ при $|\alpha|=2 k$.

Положим $r_{\omega}=2\left\|\mathscr{P}_{\omega}(0)\right\|_{C^{\gamma}\left([0, T], C^{2 k+\gamma}\right)}$. В силу оценок $(17),(21) r_{\omega} \leqslant \omega^{-(n+1)}$, где $c$ - не зависящая от $\omega$ постоянная. Оценим постоянную Липшица оператора $\mathscr{P}_{\omega}$ в шаpe $S_{\omega}:\|w\|_{C \gamma\left([0, T], C^{2 k+\gamma}\right)} \leqslant r_{w}$. Пусть $w_{1}, w_{2} \in S_{\omega}, z_{i}=\mathscr{P}_{\omega}\left(w_{i}\right), i=1,2, z=z_{2}-z_{1}$. 
Воспользуемся равенством

$$
\begin{aligned}
& z=\int_{0}^{t} d \tau \int_{\mathbb{R}^{m}} G_{w_{2}}(t, \tau, x, \xi) \\
& \times\left(\sum _ { | \alpha | = 2 k } \left[\int_{0}^{1} d s\left(\mathscr{D}_{e} a_{\alpha}\right)\left(\xi, \delta^{2 k-1} \stackrel{n}{\prime}^{\prime}+\delta^{2 k-1}\left(s w_{2}+(1-s) w_{1}\right), \tau, \omega \tau\right)\right.\right. \\
& \times\left(\delta^{2 k-1} w\right) \mathscr{D}^{\alpha}\left(\stackrel{n}{u^{\prime}}-\stackrel{0}{v}-z_{1}\right) \\
& +\left[\int_{0}^{1} d s\left(\mathscr{D}_{e} a_{\alpha}\right)\left(\xi, \delta^{2 k-1} u^{\prime}+\delta^{2 k-1}\left(s w_{2}+(1-s) w_{1}\right), \tau, \omega \tau\right)\right. \\
& \left.-\left(\mathscr{D}_{e} a_{\alpha}\right)\left(\xi, \delta^{2 k-1} \stackrel{0}{v}, \tau, \omega \tau\right)\right]\left(\delta^{2 k-1} w\right) \mathscr{D}^{\alpha} \stackrel{0}{v} \\
& \left.+\left[\left(\mathscr{D}_{e} a_{\alpha}\right)\left(\xi, \delta^{2 k-1}{ }_{v}^{0}, \tau, \omega \tau\right)-\left(\mathscr{D}_{e} \stackrel{0}{a}_{\alpha}\right)\left(\xi, \delta^{2 k-1} v_{v}^{0}, \tau\right)\right]\left(\delta^{2 k-1} w\right) \mathscr{D}^{\alpha}{ }_{v}^{0}\right] \\
& +\int_{0}^{1} d s\left(\mathscr{D}_{e} f\right)\left(\xi, \delta^{2 k-1}{ }^{n}{ }^{\prime}+\delta^{2 k-1}\left(s w_{2}+(1-s) w_{1}\right), \tau, \omega \tau\right) \\
& -\left(\mathscr{D}_{e} f\right)\left(\xi, \delta^{2 k-1}{ }_{v}^{0}, \tau, \omega \tau\right)\left(\delta^{2 k-1} w\right) \\
& \left.+\left[\left(\mathscr{D}_{e} f\right)\left(\xi, \delta^{2 k-1}{ }_{v}^{0}, \tau, \omega \tau\right)-\left(\mathscr{D}_{e} f\right)\left(\xi, \delta^{2 k-1}{ }^{0}, \tau\right)\right]\left(\delta^{2 k-1} w\right)\right) d \xi
\end{aligned}
$$

Из оценки (21) для любого $\gamma^{\prime} \in(0,1)$ следует неравенство

$$
\left\|z_{1}\right\|_{C \gamma^{\prime} /(2 k)\left([0, T], C^{\left.2 k+\gamma^{\prime}\right)}\right.} \leqslant c
$$

где $c$ - не зависящая от $\omega$ постоянная. Из этого неравенства и оценок $(20),(21)$, с помощью обычно используемой методики при оценке тепловых потенциалов типа (22) (cм., например, [2]), вьводится неравенство

$$
\|z\|_{C^{\gamma /(2 k)}\left([0, T], C^{2 k+\gamma}\right)} \leqslant c(\omega)\|w\|_{C^{\gamma /(2 k)}\left([0, T], C^{2 k+\gamma}\right)},
$$

где $\lim _{\omega \rightarrow \infty} c(\omega)=0$. Подбирая теперь $\omega_{0}>0$ так, что при $\omega>\omega_{0} c(\omega)<1 / 2$, на основании принципа сжатых отображений придем к неравенству

$$
\left\|u_{\omega}-\stackrel{n}{u}_{\omega}^{\prime}\right\|_{C^{\gamma /(2 k)}\left([0, T], C^{2 k+\gamma}\right)} \leqslant c \omega^{-(n+1)}, \quad c=\text { const, } \omega>\omega_{0}
$$

Для получения оценок погрешности в старших нормах перепишем задачу (17), (18) в виде

$$
\begin{aligned}
\frac{\partial w}{\partial t} & =\sum_{|\alpha|=2 k} a_{\alpha}\left(x, \delta^{2 k-1}\left(\stackrel{n}{ }^{\prime}+w\right), t, \omega t\right) \mathscr{D}^{\alpha} w-\sum_{|\beta| \leqslant 2 k-1} b_{\beta}(x, t, \omega t) \mathscr{D}^{2 k-1} w \\
& =\omega^{-(n+1)} a(x, t, \omega t, \omega), \quad w(x, 0)=0,
\end{aligned}
$$


где

$$
\begin{aligned}
\sum_{|\beta| \leqslant 2 k-1} b_{\beta}(x, t, \omega t) \mathscr{D}^{\beta} w:= & \sum_{|\alpha|=2 k} \int_{0}^{1}\left[\left(\mathscr{D}_{e} a_{\alpha}\right)\left(x, \delta^{2 k-1} \stackrel{n}{u}^{\prime}+s \delta^{2 k-1} w, \omega t\right)\right] d s\left(\delta^{2 k-1} w\right) \mathscr{D}^{\alpha} \stackrel{n}{\prime}^{\prime} \\
& +\int_{0}^{1}\left[\left(\mathscr{D}_{e} f\right)\left(x, \delta^{2 k-1} \stackrel{u}{\prime}^{\prime}+s \delta^{2 k-1} w, \omega t\right)\right] d s\left(\delta^{2 k-1} w\right) .
\end{aligned}
$$

Оценки $w$ по норме $C^{0}\left([0, T], C^{r}\right)$ при всех $r \geqslant 0$ получаем с помощью многократного дифференцирования задачи $(23),(24)$ по $x$ и применения неравенств (19) и (21) при $h^{1}=0$. Тогда оценки этой функции по норме $C^{s}\left([0, T], C^{r}\right), r \geqslant 0, s \geqslant 0$, получаются, если многократно продифференцировать уравнение (23) по $x, t$ и выразить из полученного уравнения производную, имеющую наибольший порядок дифференцирования по $t$. Теорема 2 доказана.

\section{СПИСОК ЦИТИРОВАННОЙ ЛИТЕРАТУРЫ}

[1] Левенштам В. Б. Экспоненциальная дихотомия и метод усреднения для параболических уравнений с быстро осциллирующей главной частью // Докл. АН СССР. 1991. Т. 318. №6. C. $1316-1319$.

[2] Левенштам В.Б.Усреднение квазилинейных параболических уравнений с быстро осциллирующей главной частью. Экспоненциальная дихотомия // Изв. РАН. Сер. матем. 1992. T. 56. № 4. C. 813-852.

[3] Симоненко И. Б. Метод усреднения в теории нелинейных уравнений параболического типа с приложением к задачам гидродинамической устойчивости. Ростов-на-Дону: Изд-во РГУ, 1989.

[4] Васильева А. Б., Бутузов В. Ф. Асимптотические разложения решений сингулярно возмущенных уравнений. М.: Наука, 1973.

[5] Васильева А. Б., Бутузов В. Ф. Асимптотические методы в теории сингулярных возмущений. М.: Высшая школа, 1990.

[6] Эйдельман С. Д. Параболические системы. М.: Наука, 1964.

[7] Стрыгин В.В.Об одной модификации метода усреднения при отыскании высшшх приближений // Прикл. матем. и механика. 1984. Т. 48. №6. С. 1042-1045.

[8] Мовлянкулов Х. Т., Шарова Л.В.Алгоритм построения высших приближений для систем с быстрыми и медленньми переменными // Дифференц. уравнения. 1986. Т. 22. № 5 . C. $755-780$.

[9] Bensoussan A., Lions J.-L., Papanicolaou G. Asymptotic Analysis for Periodic Structures. Stud. Math. Appl. V. 5. Amsterdam: North-Holland, 1978.

[10] Бахвалов Н. С., Панасенко Г. П. Осреднение процессов в периодических структурах. М.: Наука, 1984.

[11] Санчес-Паленсия Э. Неоднородные среды и теория колебаний. М.: Мир, 1984.

[12] Ж Киков В. В., Козлов С. М., Олейник О. А. Усреднение дифференциальных операторов. М.: Физматлит, 1993. 